\title{
Estudos isotópicos em águas subterrâneas do Distrito Federal: subsídios ao modelo conceitual de fluxo
}

\author{
Enéas Oliveira Lousada ${ }^{1}$ \& José Elói Guimarães Campos²
}

\begin{abstract}
Resumo Este trabalho se refere a estudos isotópicos para caracterização de um modelo de fluxo proposto para o Distrito Federal. O "Modelo de Duas Superfícies Potenciométricas" foi proposto por Campos \& FreitasSilva (1998) e estudado por outros autores. O principal objetivo é aplicar métodos hidroquímicos utilizando-se ${ }^{18} \mathrm{O},{ }^{3} \mathrm{H}$ e CFC para datar águas subterrâneas. Realizaram-se análises em amostras de águas rasas e profundas em três pontos na bacia do rio Jardim. Para a referida região, os valores $\boldsymbol{\delta}^{18} \mathrm{O}$ variam entre $-5,71 \%$ e $-5,19 \%$, os quais equivalem à média global do vapor atmosférico e precipitação nos oceanos. Portanto avalia-se que as águas subterrâneas analisadas não sofreram processos significativos capazes de modificar a composição isotópica. Os valores de trítio (UT) apresentaram-se relativamente elevados para águas rasas, indicando idades mais jovens. As águas do fraturado apresentaram valores mais baixos, associadas a épocas de recargas mais antigas. A avaliação por CFC-11 e CFC-12 corroborou as interpretações com trítio, porém possibilitou uma abordagem quantitativa. Em análises do CFC-12 para o primeiro ponto amostrado (EL1-fraturado e EL2-poroso) obtiveram-se idades aproximadas, associadas ao período de 1990 a 1997 . No segundo ponto, as amostras EL3 (fraturado) e EL4 (poroso), apresentaram variação temporal de 20 anos entre as águas analisadas, associadas ao período de 1969 a 1989, respectivamente. As amostras EL5 (poroso) e EL6 (fraturado) também mostraram valores com variação em intervalo de 15 anos para épocas de entradas das águas de precipitação nos aquíferos, associados ao período de 1974 e 1989. O resultado das análises isotópicas confirma a aplicação do modelo hidrogeológico considerado, pois os dois meios avaliados mostram intervalos de tempo que variam de 15 a 20 anos para a infiltração das águas. Esse resultado converge para a existência de dois níveis potenciométricos distintos, uma vez que o retardo para a transferência das águas dos aquíferos rasos para os profundos determina a significativa diferença de idades entre os diferentes meios.
\end{abstract}

Palavras-chave: isótopos, água subterrânea, modelo de fluxo.

\begin{abstract}
Hydrochemical studies in ground water of the Federal District: subsidies to the flow conceptual model. This paper refers to hydrochemical studies for a flow model characterization proposed to the Federal District region, Brazil. The "Two Potenciometric Surfaces Model" has been proposed by Campos \& FreitasSilva (1988) and studied by other authors. Apply hydrochemical analysis of ${ }^{18} \mathrm{O},{ }^{3} \mathrm{H}$ e $\mathrm{CFC}$ to groundwater dating is the main goal. Isotopic analyses of ${ }^{18} \mathrm{O},{ }^{3} \mathrm{H}$ and gases $\mathrm{CFC}$ has been developed in shallow and deep groundwater samples for tree points located on Jardim River watershed. The $\delta^{18} \mathrm{O}$ values vary from $-5,71 \%$ to $-5,19 \%$, which is related to global mean atmospheric vapor and ocean rains, it shows that the groundwater was not submitted to significant process able to modify the isotopic composition. The values in tritium units (UT) were relatively high for shallow groundwater, indicating young ages. Groundwater in fracture flow showed low values, indicating old recharge. The CFC-11 e CFC-12 values corroborate the tritium interpretations, and turn feasible the quantitatively evaluation. Analyzing CFC-12 for the first point (EL1 - fracture aquifer and EL2 - shallow groundwater) was obtained similar ages, associated to 1990-1997 period. For the second point, the sample EL3 (fracture) and EL4 (porous), showed temporal variation of 20 years, associated to 1969-1989 period respectively. The values in EL5 (porous) and EL6 (fractured) samples, showed 15 years interval for groundwater entrance in an aquifer, related to 1974-1989 period. The results corroborate the groundwater flow model, considering that two available places exposed time interval between 15 and 20 years for water infiltration. This result converges for an existence of two distinct potenciometric levels, considering the significant age interval related to the required time to shallow groundwater infiltrate to deeper aquifers.
\end{abstract}

Keywords: isotopes, groundwater, flow model.

INTRODUÇÃO No Distrito Federal podem ser diferenciados dois grupos de aquíferos pertencentes aos Domínios Intergranular e Fraturado. O primeiro foi subdividido em quatro sistemas $\mathbf{P}_{1}, \mathbf{P}_{2}, \mathbf{P}_{3}$ e $\mathbf{P}_{4}$ e é representado no Distrito Federal pelos solos e mantos de alteração das rochas. Nos domínios rochosos a

1 - Departamento de Ciências da Natureza, Universidade Federal do Piauí, Campus Cinobelina Elvas, Bom Jesus (PI), Brasil. E-mail: eneas@ufpi.br

2 - Instituto de Geociências, Universidade de Brasília, Campus Universitário Darcy Ribeiro, Asa Norte, Brasília (DF), Brasil. E-mail: eloi@unb.br 
água subterrânea está armazenada em fraturas, falhas e juntas, caracterizando o meio de porosidade secundária. Para o Distrito Federal, esse domínio aquífero foi dividido por Campos \& Freitas-Silva (1998) em quatro sistemas relacionados à geologia: os sistemas aquíferos Paranoá, Canastra, Bambuí e Araxá. No mesmo contexto, o sistema Paranoá foi subdividido nos subsistemas $\mathbf{S} / \mathbf{A}, \mathbf{A}, \mathbf{Q}_{3} / \mathbf{R}_{3}, \mathbf{R}_{4}$ e PPC; e o sistema Canastra nos subsistemas $\mathbf{F}$ e $\mathbf{F} / \mathbf{Q} / \mathbf{M}$.

Campos \& Freitas-Silva (1998) propuseram o "Modelo de Duas Superfícies Potenciométricas" para região do Distrito Federal, o qual foi abordado em trabalhos posteriores por Lousada (1999), Campos \& Tröger (2000), Souza (2001) e Cadamuro (2002). O modelo considera que existe uma zona não saturada entre a base do domínio intergranular e o topo da zona representada pelas fraturas saturadas. É aplicável em áreas no interior das chapadas elevadas, caracterizadas pela presença de solos bem desenvolvidos com regolitos que atingem profundidades de até $50 \mathrm{~m}$ sobre rochas intensamente fraturadas com elevado grau de interconexão entre as fraturas.

A aplicação de estudos isotópicos em pesquisas hidrogeológicas remonta há mais de 50 anos, tendo-se iniciado com os trabalhos de Urey et al. e Epstein \& Mayeda na década de 50 (Clark \& Fritz 1997). No contexto hidrológico e hidrogeológico os isótopos ambientais são utilizados como traçadores de águas superficiais e subterrâneas, em estudos de proveniência, localização da área de recarga e determinação do tempo da recarga (Clark \& Fritz 1997). Classicamente, os padrões de fluxo são determinados a partir de pontos onde se mede o nível piezométrico da água e as transmissividades são estimadas a partir de ensaios de bombeamento, sem que se tenha evidência direta do tipo, origem e idades da água. A hidrologia isotópica preenche parcialmente esta lacuna e os isótopos ambientais são particularmente indicados, pois representam os melhores traçadores uma vez que, no caso do ${ }^{3} \mathrm{H},{ }^{2} \mathrm{H} \mathrm{e}{ }^{18} \mathrm{O}$ constituem as próprias moléculas de água.

De modo geral, a maioria das pesquisas desenvolvidas com base em dados isotópicos aplicados à hidrogeologia utiliza ${ }^{18} \mathrm{O}$ para obter informações sobre as áreas de recarga e processos de fracionamento e ${ }^{14} \mathrm{C}$ para determinar a idade das águas subterrâneas antigas. Atualmente, utilizam-se largamente técnicas isotópicas para avaliação de águas jovens associadas ao ${ }^{3} \mathrm{H}$ (trítio) e ao grupo dos gases CFC (Cloro-Flúor-Carbono). Ambos utilizam curvas de concentração padrão desses elementos na atmosfera em várias regiões do planeta. A análise é realizada através de métodos comparativos entre as concentrações iniciais e atuais e marca a época em que se encerrou o contato da água com o meio atmosférico.

O objetivo deste artigo é mostrar a aplicação de métodos isotópicos para os elementos ${ }^{18} \mathrm{O},{ }^{3} \mathrm{H}$, além dos compostos de gases CFC's na datação das águas, definir as áreas de recarga e discriminar sobre eficiência e direção de fluxo, para corroborar um dos modelos hidrogeológicos conceituais proposto para a região do Distrito Federal.

\section{SÍNTESE METODOLÓGICA E SISTEMÁTICA}

DE COLETA Foram realizadas análises para ${ }^{18} \mathrm{O}$, ${ }^{3} \mathrm{H}$ e CFC em três pontos posicionados na bacia do rio Jardim (Fig. 1). A seleção destes locais contemplou as possíveis áreas de recarga, regiões de divisor de águas, condições topográficas e acesso às unidades de coleta. Em cada ponto de coleta obteve-se duas amostras de águas subterrâneas, sendo uma proveniente de cisterna ou piezômetro (domínio poroso) e uma de poço tubular profundo (domínio fraturado) (Fig. 2). Para a área de pesquisa foram analisadas seis amostras de águas subterrâneas coletadas em domínio poroso e fraturado. As águas rasas foram coletadas em cisternas e piezômetro com auxílio de amostrador ou através de sistema de bombeamento simplificado.

Para as análises de isótopos estáveis de oxigênio as amostras foram acondicionadas em recipientes plásticos de baixa porosidade e não reagente com volume de $200 \mathrm{ml}$. A coleta foi realizada de modo a impedir a presença de bolhas de ar no interior do recipiente, fato comum quando se utiliza bomba submersa para a extração da amostra. Nesse caso utilizou-se um outro recipiente onde foi possível a imersão total do frasco para proceder a coleta.

Para as coletas das amostras para Trítio foram utilizados frascos de vidro de $100 \mathrm{ml}$. Este recipiente foi inserido em uma lata de zinco preenchida com água da mesma fonte, a qual foi vedada. Esse procedimento é necessário para impedir trocas entre os gases das amostras e os atmosféricos, fator que pode interferir nos resultados das análises.

As coletas de águas para análises de CFC são mais simples. $\mathrm{O}$ procedimento requer um recipiente plástico de $500 \mathrm{ml}$ para acondicionamento da amostra e a coleta é realizada diretamente na fonte com auxílio de bailers (cisternas) ou bombas submersas (poço profundo).

O laboratório HYDROISOTOP, sediado na Alemanha e com reconhecimento internacional por pesquisadores da área, realizou as análises para os isótopos estáveis ${ }^{18} \mathrm{O}$ e ${ }^{2} \mathrm{H}$, isótopo radioativo ${ }^{3} \mathrm{H}$ e para os gases CFC. Os métodos laboratoriais utilizados foram: espectrometria de cintilação líquida (LSC) para o trítio $\left({ }^{3} \mathrm{H}\right)$; espectrometria de massas para determinação de razão isotópica (IRMS) para o oxigênio-18 $\left(\delta^{18} \mathrm{O}\right)$ com VSMOW-Std.: $\pm 0,15 \%$ e para o deutério $\left(\delta^{2} \mathrm{H}\right)$ com VSMOW-Std.: $\pm 1,5 \%$; e, cromatografia gasosa com detecção por captura de elétrons (GC-ECD) para os gases CFC.

\section{ESTUDO DE ISÓTOPOS ESTÁVEIS DE OXIGÊ-}

NIO A base da geoquímica de isótopos ambientais é a comparação global de dados, o que requer uma padronização das medições entre os laboratórios. Há poucas décadas, materiais apropriados foram estabelecidos como padrões ou referências isotópicas reconhecidas internacionalmente. Os dados provenientes das análises realizadas em espectrômetros de massa são emitidos referenciados a esses padrões. 


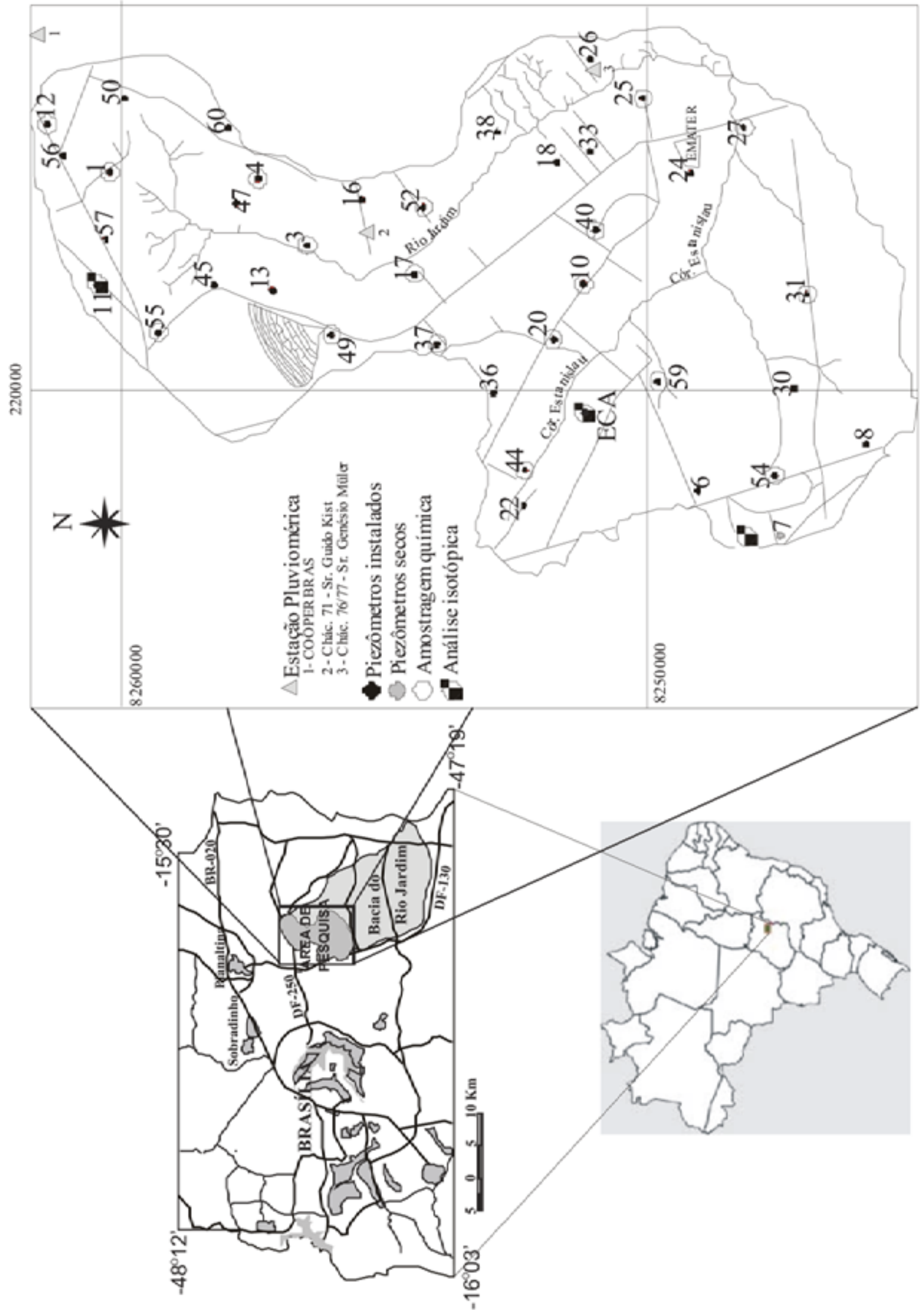




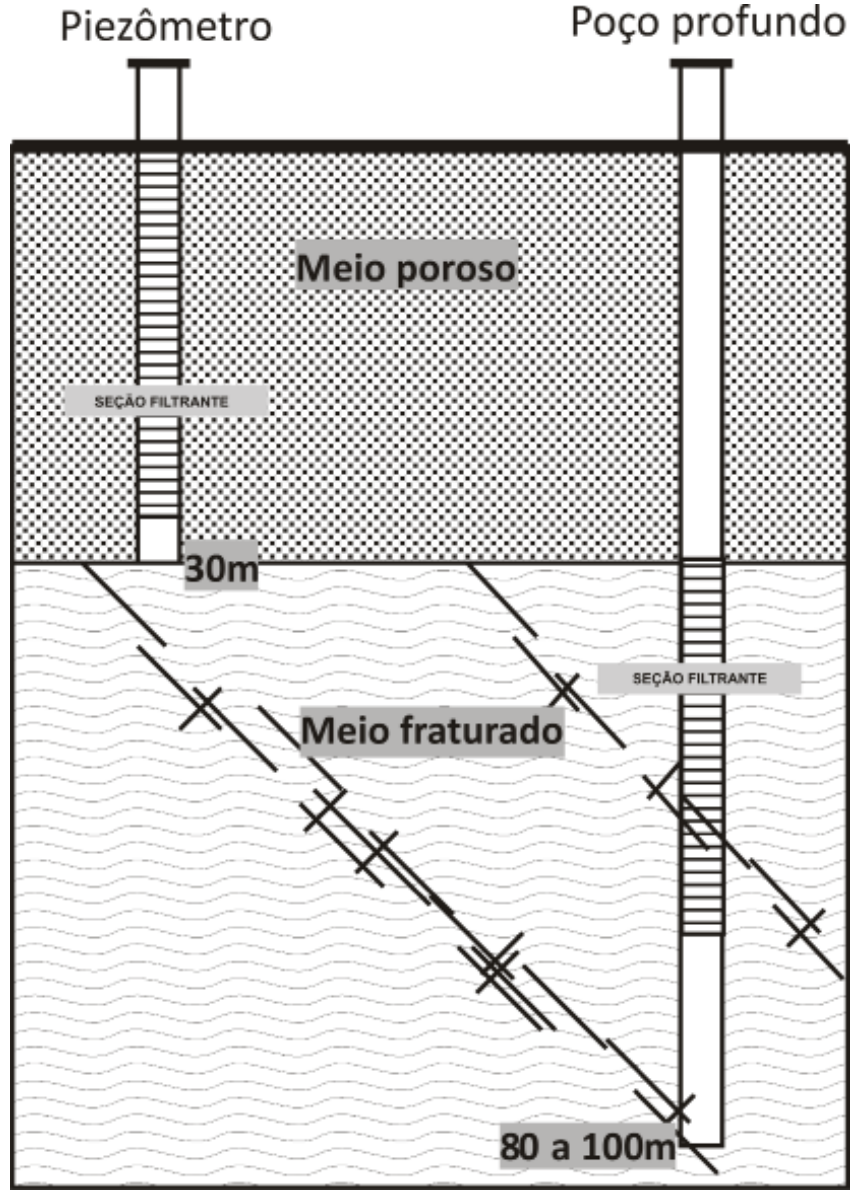

Figura 2 - Perfil construtivo dos poços de coleta para as análises isotópicas das águas. Os piezômetros foram utilizados para coletas das águas do domínio poroso e os poços profundos para as do fraturado.

Craig (1961) introduziu Standard Mean Ocean Water (SMOW) como padrão para medições de ${ }^{18} \mathrm{O}$ e ${ }^{2} \mathrm{H}$ em águas. Na realidade, este foi proveniente de uma água hipotética que teve seus conteúdos isotópicos calibrados segundo NBS-1. A amostra de água foi originária do rio Potomac e formalmente catalogada pelo National Bureau of Standards (NBS). Craig (1961) definiu SMOW como:

$\left({ }^{18} \mathrm{O} /{ }^{16} \mathrm{O}\right)_{\text {SMOW }}=1,008\left({ }^{18} \mathrm{O} /{ }^{16} \mathrm{O}\right)_{\mathrm{NBS}-1}=(1993,4 \pm 2,5) \cdot 10^{-6}$ $\left({ }^{2} \mathrm{H} /{ }^{1} \mathrm{H}\right)_{\mathrm{SMOW}}=1,050\left({ }^{2} \mathrm{H} /{ }^{1} \mathrm{H}\right)_{\mathrm{NBS}-1}=(158 \pm 2) .10^{-6}$.

Posteriormente, o IAEA (1983) preparou uma água padrão a partir da destilação de água do oceano, que foi modificada para uma composição próxima do SMOW. Essa referência é identificada como VSMOW (Vienna Standard Mean Ocean Water), que mostram:

$\left({ }^{18} \mathrm{O} /{ }^{16} \mathrm{O}\right)_{\text {VSMOW }}=(2005,2 \pm 0,45) \cdot 10^{-6}$ (Baertschi 1976) $\left({ }^{2} \mathrm{H} /{ }^{1} \mathrm{H}\right)_{\mathrm{vsmow}}=(155,76 \pm 0,05) \cdot 10^{-6}($ Hageman et al. 1970).

VSMOW vem sendo a referência internacionalmente aceita para ${ }^{18} \mathrm{O}$ e ${ }^{2} \mathrm{H}$ em águas. A partir da abundância de medidas absolutas, verificou-se que VSMOW é mais de $5 \%$ enriquecido em ${ }^{18} \mathrm{O}$ em relação ao SMOW, e $14 \%$ empobrecido em ${ }^{2} \mathrm{H}$.
Avaliações por isótopos estáveis consistem em comparar os dados da precipitação atual e os da água subterrânea que se pretende avaliar, considerando os aspectos dimensionais e temporais inerentes ao processo. Quando os dados de precipitação local não se assemelham aos da água subterrânea, não se pode afirmar que a recarga não seja local, pois alguns fatores podem modificar a composição isotópica da água antes, durante ou depois da recarga (Siqueira 1978).

Valores $\boldsymbol{\delta}^{\mathbf{1 8}} \mathbf{O}-\boldsymbol{\delta}^{\mathbf{2}} \mathbf{H}$ Para análise de isótopos estáveis as amostras foram tratadas em conjunto por possuírem valores $\boldsymbol{\delta}^{18} \mathrm{O}$ muito semelhantes. O diagrama $\boldsymbol{\delta}^{2} \mathrm{H}-\boldsymbol{\delta}^{18} \mathrm{O}$ para todas as amostras de água analisadas é apresentado na figura 3. Durante a análise dos resultados observou-se que as linhas das águas meteóricas que representam os dados da precipitação e das amostras coletadas na área em apreço, são equivalentes à linha meteórica global média (GMWL) (Fig. 3). As equações que regem as linhas abordadas são: (1) $\boldsymbol{\delta}^{2} \mathrm{H}=8,0 \boldsymbol{\delta}^{18} \mathrm{O}+10,0$, para a linha média da água meteórica global (SMOW); (2) $\boldsymbol{\delta}^{2} \mathrm{H}$ $=7,6 \boldsymbol{\delta}^{18} \mathrm{O}+10,2$, linha média para os valores da precipitação local; e, (3) $\boldsymbol{\delta}^{2} \mathrm{H}=8,3 \boldsymbol{\delta}^{18} \mathrm{O}+9,3$, para os valores obtidos a partir de análises de água subterrânea na bacia do alto Jardim. Para a referida região, os valores de $\delta^{18} \mathrm{O}$ variam entre $-5,71 \%$ e $-5,19 \%$, os quais equivalem à média global do vapor atmosférico e precipitação nos oceanos. Dessa forma, avalia-se que as águas subterrâneas analisadas não sofreram processos significativos capazes de modificarem a composição isotópica. As sutis diferenças podem estar relacionadas às altitudes e umidades nos locais de recarga, interferindo na inclinação da reta para os valores $\boldsymbol{\delta}^{2} \mathrm{H}$ locais. De modo geral, as águas analisadas são estáveis desde a origem até a penetração e movimentação no aquífero, tal fato fornece subsídio para inferir que, as precipitações locais ou em regiões próximas à bacia são responsáveis pela recarga dos mananciais subterrâneos, o que acontece de forma rápida. A distribuição espacial da composição isotópica nessa bacia é homogênea, indicando a presença de importantes fluxos subterrâneos para produzir, no aquífero, mistura e homogeneização de águas infiltradas em diferentes áreas da bacia.

DATAÇÃO COM TRÍTIO O termo idade da água subterrânea pode ter duas interpretações, podendo em alguns casos representar o tempo relativo da presença da água no aquífero ou em outros casos, o tempo absoluto em que a mistura da água passou a integrar o domínio subterrâneo após sua infiltração através da zona vadosa. O trítio é parte integrante da molécula de água e, portanto, permite "datar" mais facilmente as águas. Todos os outros métodos de datação estão relacionados aos constituintes dissolvidos, cuja abundância na água é controlada por processos físico-químicos e biológicos, e assim permitem datações relativas.

Processos como mistura hidrodinâmica e convergência do padrão de fluxo subterrâneo acarretam em variação na origem da recarga e idade da água. Somente 


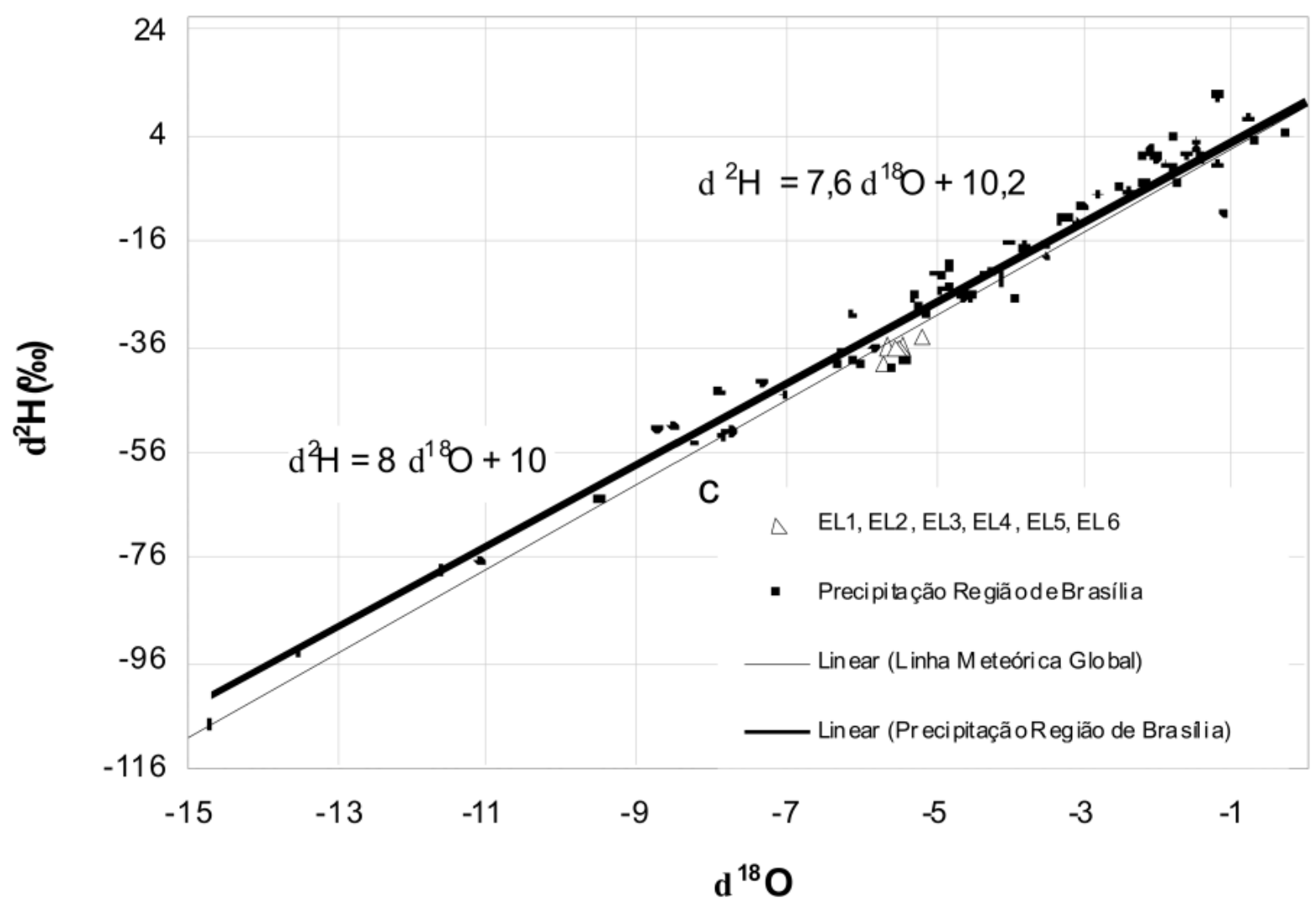

Figura 3 - Gráfico representativo das equações e linhas: global (GMWL), local elaborada a partir das amostras analisadas e da precipitação baseada nos valores da região (Estação Aeroporto).

em poços restritos e usualmente em aquíferos artesianos regionais, pode ser preservada a idade ao longo do padrão de fluxo. Como consequência, o termo "idade" passa a ser referido como "tempo médio de residência da água subterrânea".

Análises isotópicas de trítio são largamente utilizadas com eficiência para avaliarem sobre a ocorrência de recarga atual. Os testes termonucleares na atmosfera, que ocorreram de maio de 1951 a 1976, acarretaram nos sinais e no pico máximo de concentração de trítio na atmosfera, valores estes utilizados para estudo das águas subterrâneas modernas. $\mathrm{O}$ trítio $\left({ }^{3} \mathrm{H}\right)$ é um radioisótopo do hidrogênio, produzido naturalmente por radiação cósmica, com curta permanência e meia-vida de 12,43 anos (Unterweger et al. 1980). Até o início da década de 90, a maioria do trítio originado nestes testes foi lavada da atmosfera e o nível de trítio na precipitação global, atualmente, é próximo dos níveis naturais.

A geofísica do trítio tem sido um assunto intensamente pesquisado nas últimas décadas. $\mathrm{Na}$ sua essência, o método consiste em determinar a concentração de trítio nas águas de precipitação acumuladas em um determinado nível e a concentração de trítio em uma amostra proveniente deste nível (Tolskikhin \& Kamenskiy 1969).

Para avaliar os resultados obtidos para as amostras coletadas na área, tentou-se a utilização de métodos quantitativos, no entanto, a ausência de dados de longo período e de curvas padrões para o hemisfério sul restringiu o procedimento.

Outra forma de avaliação utilizando isótopos de trítio é o método de análises em séries temporais. Nesse caso são realizadas amostragens sequenciais da água subterrânea para identificação do pico de concentração máxima (associado aos testes nucleares), que fornece uma boa indicação sobre o tempo médio de residência. A aplicação desse método na área de estudo ficou restrita em decorrência do longo tempo necessário e dos elevados custos das análises para o radioisótopo trítio.

Considerando-se a impossibilidade de tratamento quantitativo para as análises de trítio, propõe-se uma avaliação qualitativa dos dados conforme preceitos teóricos abordados por Clark \& Fritz (1997).

Vários autores tratam da implementação de técnicas isotópicas para datação das águas e elucidações sobre as condições hidrogeológicas locais, quase sempre associadas às análises de ${ }^{3} \mathrm{H}-{ }^{3} \mathrm{He}$ em conjunto com outras ferramentas de datações (Tolstikhin \& Kamenskiy 1969, Schlosser et al. 1989, Eckwurzel et al. 1994, Szabo et al. 1996, Solomon et al. 1993). As abordagens quantitativas, utilizando-se as concentrações de ${ }^{3} \mathrm{H}$ (trítio), tornam-se difíceis quando não se dispõem das séries completas de concentrações nas águas de precipitações da área pesquisada.

Desde os últimos grandes testes nucleares, as concentrações de trítio na atmosfera vêm sendo atenuadas, e os níveis atuais estão basicamente associados 
às produções atmosféricas. Essa evolução da demanda de trítio, associada a misturas em alguns aquíferos com águas pré-testes nucleares, dificulta as interpretações refinadas quanto às idades das águas subterrâneas. Diante da impossibilidade de análise quantitativa sobre o tempo de residência da água subterrânea coletada na área de pesquisa, optou-se por realizar abordagens qualitativas como proposto por (Clark \& Fritz 1997).

Para regiões continentais:

$<0,8$ UT Submodernas - recarga anterior a 1952.

$0,8 \mathrm{a} \sim 4$ UT Misturas entre recargas submodernas e recentes.

5 a 15 UT Modernas ( $<5$ a 10 anos).

15 a 30 UT Algum indício de ${ }^{3} \mathrm{H}$ dos testes está presente.

> 30 UT Considerável componente de recarga entre as décadas de 60 e 70.

$>50$ UT Recarga dominantemente da década de 60.

COMPOSTOS DE CFC Os compostos de cloro-flúor-carbono (CFC) são utilizados por pesquisadores para a datação de águas juvenis, apesar da presença de tais compostos na atmosfera não ser desejável. Estes compostos são resistentes à degradação, tornando-os um excelente marcador quando aplicados para águas subterrâneas jovens. A concentração dos compostos de CFC na atmosfera vem sendo incrementada desde 1940, gerando sua curva de concentração em função da ocorrência na atmosfera ao longo dos anos (input function). Os compostos de CFC foram largamente usados para investigar sobre o padrão de circulação nos oceanos durantes as últimas décadas. Thompson \& Hayes (1979) e estudos posteriores documentaram seus usos para datação de águas subterrâneas jovens. Avaliações recentes sobre o impacto global dos compostos de CFC nas águas subterrâneas podem ser encontradas no trabalho desenvolvido por Höhener et al. (2003). Os dados da concentração dos compostos de CFC e SF6 podem ser usados para indicar o fluxo de águas juvenis (mais jovens que 50 anos) (Busenberg \& Plummer 1993, Oster et al. 1996), para tanto suas concentrações na atmosfera - função de entrada - durante os últimos 50 anos foram reconstruídas (Cunnold et al. 1997) e estudos químicos e físicos determinaram seus valores de solubilidade.

Abordagens sobre datação de águas subterrâneas aplicam o termo "idade" como sendo a data de introdução do composto químico, e não da água. A concentração de CFC pode sofrer degradação e absorção durante o deslocamento, por essa razão refere-se à "idade" como "idade modelo" ou "idade aparente". A ênfase nesses termos é necessária para simplificar abordagens em relação aos processos de transporte ou outros que possam afetar a concentração de CFC que, no caso, são desconsiderados (Clark \& Fritz 1997).

A maioria das idades aparentes CFCs referidas na literatura, bem como as abordadas nesta pesquisa, é baseada numa simplificação do modelo piston flow, onde a idade interpretada assume que a concentração de CFC não foi alterada durante o transporte do ponto de entrada ao local de medida no aquífero.

Com finalidade de datações, podem ser utilizadas três principais substâncias, abreviadas como CFC11 (triclorofluormetano $\mathrm{CFCl}_{3}$ ), $\mathrm{CFC}-12$ e CFC-113 (triclorotrifluoretano, $\mathrm{C}_{2} \mathrm{~F}_{3} \mathrm{Cl}_{3}$ ). Suas concentrações são medidas e comparadas com a curva de concentração dos CFCs nas águas de precipitação para os últimos 50 anos. Em geral, são usadas as concentrações absolutas ou razões em pares de CFCs.

$\mathrm{Na}$ bacia do rio Jardim analisaram-se 6 amostras coletadas em cisternas (EL2, EL4 e EL6) e poços profundos (EL1, EL3 e EL5), para os gases CFC11, CFC113, CFC12 (Fig. 4).

As amostras foram dispostas graficamente de acordo com suas idades. Das amostras analisadas, os pontos EL3 e EL5 apresentaram mais baixas concentrações em CFCs e trítio (0,8 e 1,4 U.T. respectivamente). Em análise conjunta dos valores para o trítio e CFCs (Fig. 4), verifica-se que as amostras EL1, EL2, EL4 e EL6 se posicionam numa região equivalente quanto às suas concentrações, sendo estas amostras mais jovens caracterizadas por concentração intermediária.

\section{O MODELO DAS DUAS SUPERFÍCIES POTEN-} CIOMÉTRICAS O modelo é aplicável em áreas no interior das chapadas elevadas, caracterizadas pela presença de solos bem desenvolvidos (latossolos), com regolitos que atingem profundidades de até $50 \mathrm{~m}$ sobre rochas intensamente fraturadas com elevado grau de interconexão entre as fraturas.

O Modelo de Duas Superfícies Potenciométricas considera que existe uma zona não saturada entre a base do domínio poroso e o topo da zona representada pelas fraturas saturadas.

É composto por dois meios aquíferos distintos, denominados aquíferos dos domínios intergranular e fraturado. Para esses diferentes meios, identificam-se duas superfícies potenciométricas: superfície potenciométrica do domínio intergranular e superfície potenciométrica do domínio fraturado (Fig. 5).

O domínio intergranular é constituído pelos solos e pela porção alterada da rocha (regolito) e sua superfície potenciométrica varia de 08 a $20 \mathrm{~m}$ de profundidade com respostas rápidas aos efeitos da precipitação. Este meio desenvolve porosidade eminentemente primária onde a água é armazenada nos espaços intergranulares. O domínio fraturado é constituído pela rocha sã, onde a ocorrência da água se restringe aos espaços originados por esforços tectônicos (fraturas), caracterizando-os como de porosidade secundária.

Os dois aquíferos são diretamente associados, no entanto, não são permanentemente conectados ao longo do ano. No período de seca, quando a descarga de base é responsável pelo rebaixamento do nível d'água do aquífero poroso, eles podem ser isolados um do outro, sendo verificada apenas a porção não saturada e saturada do meio fraturado.

O topo do aquífero fraturado é coincidente ao dos metassedimentos frescos, onde os planos de 


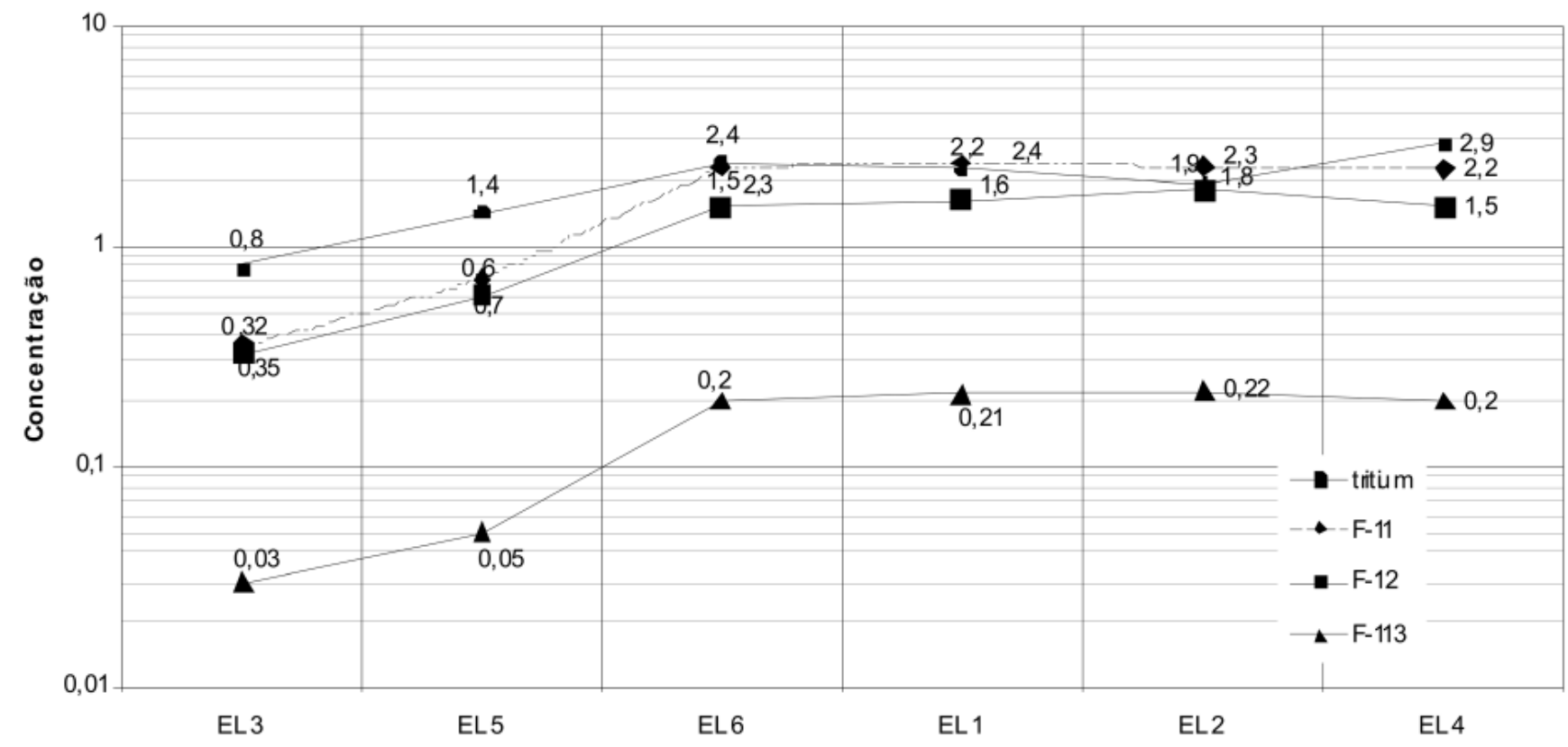

Figura 4 - Tratamento integrado dos gases analisados (CFC-11, CFC-12, CFC-113 e trítio) para as amostras EL1, EL3, EL5 (aquifero fissural) e EL2, EL4, EL6 (aquifero poroso) em ordem crescente para os valores que indicam as idades da recarga. O trítio é dado em UT (Unidades de Trítio) e os CFCs em pmol/l.

descontinuidades como juntas e falhas compõem os reservatórios. No limite entre a rocha fraturada e o saprolito há estreitamento da abertura das fraturas em função do acúmulo de argilas que migram por iluviação. No período em que a carga hidráulica no aquífero poroso não é suficiente para quebrar a barreira hidráulica estabelecida, o topo do meio fraturado apresenta-se não saturado. As fraturas podem permanecer secas ou parcialmente saturadas até profundidades que variam entre 50 e $75 \mathrm{~m}$ (superfície potenciométrica do domínio fraturado). A partir deste patamar as fraturas são permanentemente saturadas por água.

A barreira hidráulica, presente na interface dos dois aqǘferos, não permite o fluxo contínuo por

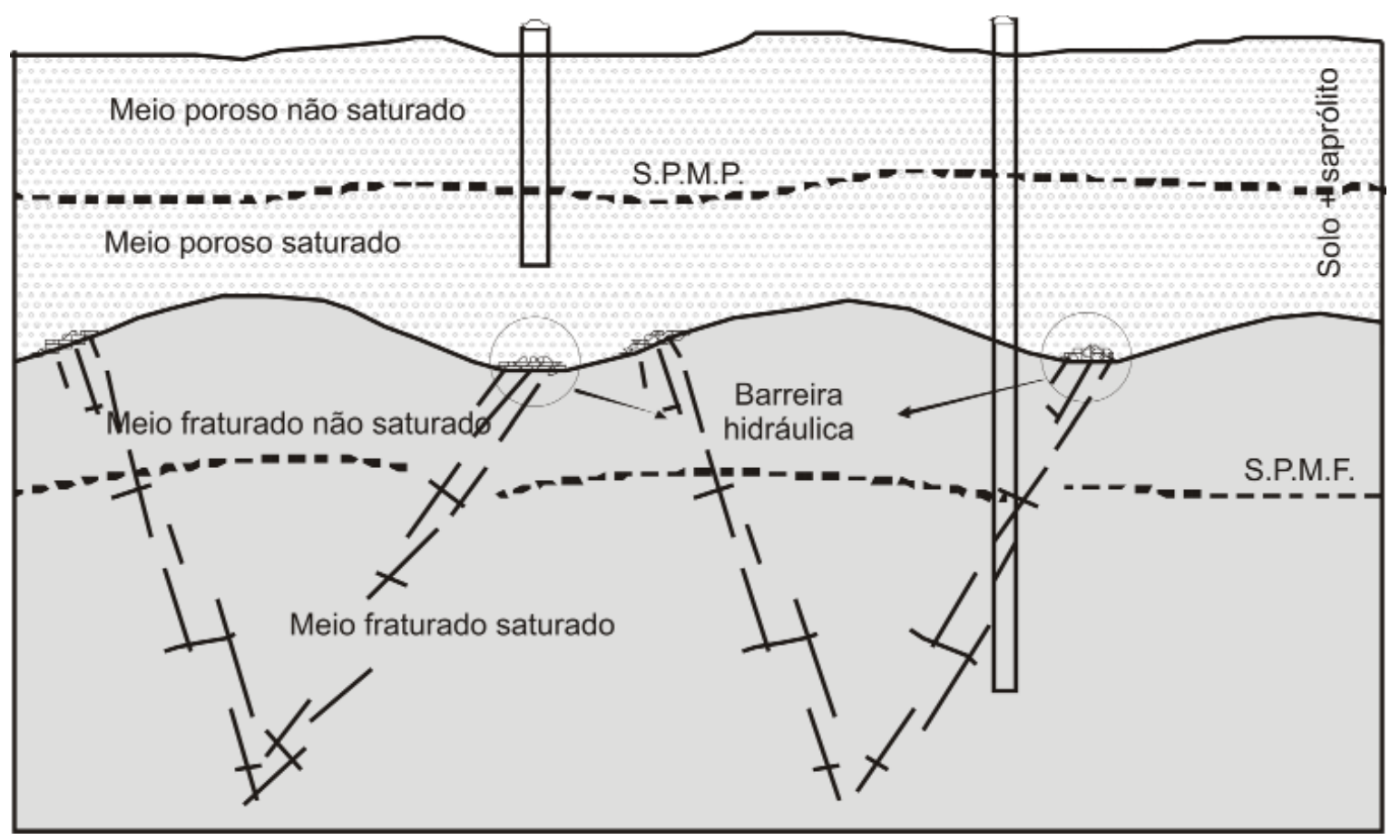

Figura 5 - Esquema do modelo das duas superficies potenciométricas. S.P.M.P. - Superficie Potenciométrica do Meio Poroso, S.P.M.F. - Superficie Potenciométrica do Meio Fraturado. 
gotejamento entre estes. Essa barreira é constituída por material fino (argila e silte) que limita a abertura das fraturas, por forças capilares e pela presença de ar na porosidade planar secundária. A anisotropia do sistema fraturado também auxilia no desenvolvimento da barreira hidráulica. Pode-se afirmar que se trata de uma barreira capilar.

A recarga do meio fraturado se dá após uma elevação da carga hidráulica no meio superior que permita a ruptura da barreira hidráulica e a passagem para as fraturas na rocha. Nos meses mais chuvosos do ano, quando o nível freático alcança a máxima elevação, pode haver a junção das duas zonas saturadas, entretanto, na maior parte do ano, há a separação por uma zona de fraturas não saturada.

DISCUSSÃO DOS RESULTADOS A tabela 1 apresenta os valores de CFCs, trítio, $\delta^{18} \mathrm{O}$ e deutério obtidos para as amostras analisadas.

Datações com Trítio $\left({ }^{3} \mathbf{H}\right) \quad$ Em análise, observou-se que as amostras EL1, EL2, EL4 e EL6 apresentaram valores relativamente elevados, resultando em águas mais jovens e, as EL3 e EL5, com valores mais baixos, estão associadas a épocas de recargas mais antigas.

Para as amostras EL3 e EL4, obteve-se uma diferença de 2,1 UT. Para esse par, avalia-se que a amostra EL3 (0,8 UT), coletada em poço tubular profundo que intercepta fraturas a profundidades maiores que $50 \mathrm{~m}$, é submoderna (Clark \& Fritz 1997), associada ao período pré-testes nucleares (anterior a 1952). Para esse ponto pode-se inferir como o de idade menos jovem dentre os outros analisados, onde as águas penetraram no aquífero há mais de quatro décadas. A amostra EL4 (2,9 UT), adquirida para o meio poroso no mesmo local onde se coletou a amostra EL3, é associada à época onde predomina recarga recente.

Para o par EL5 e EL6, obteve-se intervalo de 1 UT e os valores obtidos para as duas amostras sugerem recarga recente.
Em síntese, avalia-se que a presença de trítio $\left({ }^{3} \mathrm{H}\right)$ em todas as amostras coletadas resulta em idade recente para as águas, onde as entradas nos aquíferos podem estar associadas a épocas que variam desde o início da década de 50 aos tempos atuais.

Datações com Clorofluorcarbonos (CFCs) O método de datação com CFCs é baseado em comparações com curvas padrões para as concentrações dos elementos nos hemisférios norte e sul. Essas curvas são obtidas pela aquisição de medidas periódicas ao longo do tempo em várias estações monitoradas pela National Oceanic and Atmospheric Administration (NOAA). Para o hemisfério sul as estações disponíveis são Cape Matula (Samoa) e Cape Grim (Tasmânia) (Cunnold et al. 1997), sendo estas utilizadas para a avaliação dos dados da bacia do rio Jardim.

$\mathrm{O}$ método para tratamento dos dados obtidos teve como base os CFCs 11 e 12 por possuírem séries mais completas.

Foram feitas análises individualizadas para o CFC11 e CFC12, relacionando as idades com suas concentrações quando solúveis em água.

Em avaliação subsidiada pelo CFC11, obteve-se que as amostras EL1, EL2, EL4 e EL6 são mais jovens e as EL3 e EL5 mais antigas, corroborando as interpretações com o trítio. Para o par EL1 e EL2 obteve-se idades muito aproximadas, associadas ao período de 1986 a 1985. Para o par EL3 e EL4, obteve-se variação temporal de 16 anos entre as águas analisadas, associadas ao período de 1968 a 1984. As amostras EL5 e EL6 também mostraram valores com variação em um intervalo de 13 anos para as épocas de entradas das águas de precipitação nos aquíferos, associados ao período de 1972 e 1985. Esses valores são coerentes e fornecem fortes subsídios quando integrados às análises com trítio, para analisar sobre o tempo de residência médio das águas nos aquíferos.

As informações resultantes das análises de $\mathrm{CFC} 12$ foram semelhantes às $\mathrm{CFC} 11$, porém resultaram em idades mais recentes. Para as amostras EL1-EL2 obteve-se

Tabela 1 - Resumo dos valores obtidos para as análises isotópicas de $C F C$, oxigênio- $18\left(\delta^{18} O\right)$, deutério $\left(\delta^{2} H\right) e$ trítio $\left.{ }^{\beta} H\right)$. EL1, EL3 e EL5 - águas provenientes de aquiferos fraturados. EL2, EL4 e EL6 - águas provenientes de aquiferos porosos. ${ }^{3} \mathrm{H}$ em unidades de trítio (TU) com duplo desvio padrão (1 UT $\left.=0,119 \mathrm{~Bq} / \mathrm{l}\right) . \delta^{18} \mathrm{O}$ relacionado ao VSMOWstd.: $\pm 0,15 \delta^{18}$ O VSMOW-\%o.

\begin{tabular}{lccccccc}
\hline \multirow{2}{*}{ PARÂMETRO } & \multirow{2}{*}{ UNID } & \multicolumn{5}{c}{ AMOSTRAS } \\
\cline { 3 - 8 } & & EL-1 & EL-2 & EL-3 & EL-4 & EL-5 & EL-6 \\
\hline Triclorofluormetano $(\mathrm{F} 11)$ & $\mathrm{pmol} / 1$ & $2,4 \pm 0,3$ & $2,3 \pm 0,3$ & $0,35 \pm 0,05$ & $2,2 \pm 0,3$ & $0,7 \pm 0,1$ & $2,3 \pm 0,3$ \\
\hline Diclorodifluormetano $(\mathrm{F} 12)$ & $\mathrm{pmol} / 1$ & $1,6 \pm 0,2$ & $1,8 \pm 0,2$ & $0,32 \pm 0,05$ & $1,5 \pm 0,2$ & $0,6 \pm 0,2$ & $1,5 \pm 0,2$ \\
\hline $1,1,2$-Triclorotrifluoretano $(\mathrm{F} 113)$ & $\mathrm{pmol} / 1$ & $0,21 \pm 0,05$ & $0,22 \pm 0,05$ & $0,03 \pm 0,05$ & $0,2 \pm 0,05$ & $0,05 \pm 0,05$ & $0,2 \pm 0,05$ \\
\hline Oxigênio-18 $\left(\delta^{18} \mathrm{O}\right)$ & $\%$ & $-5,43$ & $-5,48$ & $-5,71$ & $-5,63$ & $-5,51$ & $-5,19$ \\
\hline Deutério $\left(\delta^{2} \mathrm{H}\right)$ & $\%$ & $-36,2$ & $-36,5$ & $-39,3$ & $-35,8$ & $-36,4$ & $-33,8$ \\
\hline Excesso de Deutério & $\%$ & 7,2 & 7,3 & 6,4 & 9,2 & 7,7 & 7,7 \\
\hline Trítio $\left({ }^{3} \mathrm{H}\right)$ & $\mathrm{UT}$ & $2,2 \pm 0,8$ & $1,9 \pm 0,9$ & $0,8 \pm 0,8$ & $2,9 \pm 1,0$ & $1,4 \pm 1,1$ & $2,4 \pm 0,8$ \\
\hline
\end{tabular}


idades associadas aos anos de 1990,5 e 1997,5 respectivamente. Nessa avaliação esse ponto mostrou diferença significativa entre as idades para as águas dos meios poroso e fraturado, sendo de aproximadamente 7 anos. Para o par EL3 e EL4, obteve-se intervalo de tempo de aproximadamente 20 anos, associando-se as águas do meio fraturado ao período de 1969,5 e as do meio poroso ao de 1989,5. As amostras EL5 e EL6 apresentaram intervalo de 15 anos, sendo o ano de 1974,5 estipulado para a água coletada em meio fraturado ter cessado contato com a atmosfera, e, o ano de 1989,5, para as águas do meio poroso passarem pelo mesmo processo.

A tabela 2 reúne os valores obtidos para CFC11 e CFC12 e idades avaliadas com base na curva padrão para as concentrações dos elementos quando solúveis em água.

A apresentação gráfica integrada para concentração de CFCs 11 e 12 gera curvas onde constam os padrões de concentração para a concentração destes gases quando solúveis em água e os pontos relacionados às idades das amostras analisadas (Fig. 6). Nessa avaliação se observa diretamente a relação entre os padrões de concentrações mundiais e os pontos locais avaliados. De modo geral, as avaliações mostraram idades mais recentes para as amostras EL1, EL2, EL4 e EL6, e, mais antigas para as amostras EL3 e EL5, ambas representativas do meio fraturado. Nesta avaliação gráfica observa-se ainda a equivalência entre as idades das amostras EL2 e EL6, para o CFC-11 e EL4 e EL6, para o CFC-12.

Esses dados confirmam que as condições hidrogeológicas e isotópicas são distintas para os diferentes meios aquíferos. Os mecanismos de transmissão das águas do poroso para as fraturas na rocha atuam em padrões de circulação de águas jovens, considerando-se que todas as amostras mantiveram contato com a atmosfera posteriormente ao período 1930-1940, quando iniciaram as primeiras emissões de CFCs. Os tempos de residência das águas em domínio poroso e fraturado mostram que estes aquíferos possuem dinâmicas diferentes, considerando-se que os mecanismos de fluxo subterrâneo desde as áreas de recarga até os aquíferos avaliados ocorreram em diferentes condições temporais,

Tabela 2 - Valores obtidos para CFC11 e CFC12 (pmol/l) e as idades avaliadas com base nas curvas padrão para as concentrações dos elementos quando solúveis em água.

\begin{tabular}{lcccc}
\hline Amostra & $\begin{array}{c}\text { F11 } \\
(\mathbf{p m o l} / \mathbf{l})\end{array}$ & $\begin{array}{c}\text { Ano de } \\
\text { recarga }\end{array}$ & $\begin{array}{c}\text { F12 } \\
(\mathbf{p m o l} / \mathbf{l})\end{array}$ & $\begin{array}{c}\text { Ano de } \\
\text { recarga }\end{array}$ \\
\hline EL1 & 2,4 & 1986 & 1,6 & 1990,5 \\
\hline EL2 & 2,3 & 1985 & 1,8 & 1997,5 \\
\hline EL3 & 0,35 & 1968 & 0,32 & 1969,5 \\
\hline EL4 & 2,2 & 1984 & 1,5 & 1989,5 \\
\hline EL5 & 0,7 & 1972 & 0,6 & 1974,5 \\
\hline EL6 & 2,3 & 1985 & 1,5 & 1989,5 \\
\hline
\end{tabular}

resultando em intervalos de 15 e 20 anos. Esse resultado corrobora as abordagens sobre os modelos conceituais em que se referem à existência de duas superfícies potenciométricas distintas para cada meio aquífero.

Modelo conceitual de fluxo Os resultados convergem para a existência de dois níveis potenciométricos distintos, uma vez que o retardo para a transferência das águas dos aquíferos rasos para os profundos inferiu em significativa diferença de idades entre os diferentes meios. A água que infiltra no meio poroso é relativamente jovem, como se verificou nas análises de trítio e CFCs e corroborada pelos isótopos estáveis de oxigênio. O mecanismo de transmissão das águas em meio intergranular para o meio fraturado ocorre lentamente em condições de fluxo vertical e recarga local, ou, quando provenientes de recarga em áreas adjacentes, assumem fluxo condicionado pelas condições de fraturamento da rocha, no que se refere à abertura e interconexão. $\mathrm{O}$ fluxo em meio poroso é condicionado basicamente pelo gradiente hidráulico atuante, onde parte da água infiltrada segue por fluxo de base e alimenta as drenagens superficiais e, outra porção, é transmitida para as fraturas. Em meio fraturado o fluxo é associado às descontinuidades planares abertas. Nas estruturas verticais o fluxo é eficiente, por outro lado, as fraturas sub-horizontais condicionam fluxo mais lento, corroborado ainda pela deficiência no que se refere à presença de exutórios.

CONCLUSÕES O Modelo das Duas Superfícies Potenciométricas é aplicável em áreas do Distrito Federal e foi corroborado pela idade das águas subterrâneas dos meios intergranular e fraturado, que apresentaram uma diferença de 15 e 20 anos. O referido modelo é subsidiado ainda por dados provenientes da construção e testes de produção de poços tubulares profundos, pelo monitoramento da descarga e qualidade das águas de nascentes, pela avaliação do comportamento de balanços hídricos em pequenas bacias hidrográficas e monitoramento da variação dos níveis freáticos em diferentes aquíferos.

A presente pesquisa resultou em fortes subsídios para a discriminação dos padrões de circulação da água subterrânea, aplicando-se estudos hidrogeológicos e análises isotópicas. Considera-se que foi uma iniciativa pioneira com resultados inéditos para a região do Distrito Federal, em que os dados obtidos poderão ser utilizados como parâmetros de avaliação quando comparados a regiões de mesmas características geológicas, hidrogeológicas e estruturais.

Para avaliações hidrogeológicas baseadas em química isotópica, sugere-se que estas sejam realizadas em completo domínio quanto às condições nos locais de coleta. Para amostragem em poços tubulares profundos é extremamente relevante o conhecimento das características construtivas das obras, portanto, a condição ideal é a construção de unidades especificas para tal finalidade.

Na medida do possível deve-se investir em análises de trítio e seu isótopo filho (o hélio tritiogênico) além de intensificar as avaliações com uso dos CFCs 
CFC-11 e CFC-12 -Bacia Rio Jardim

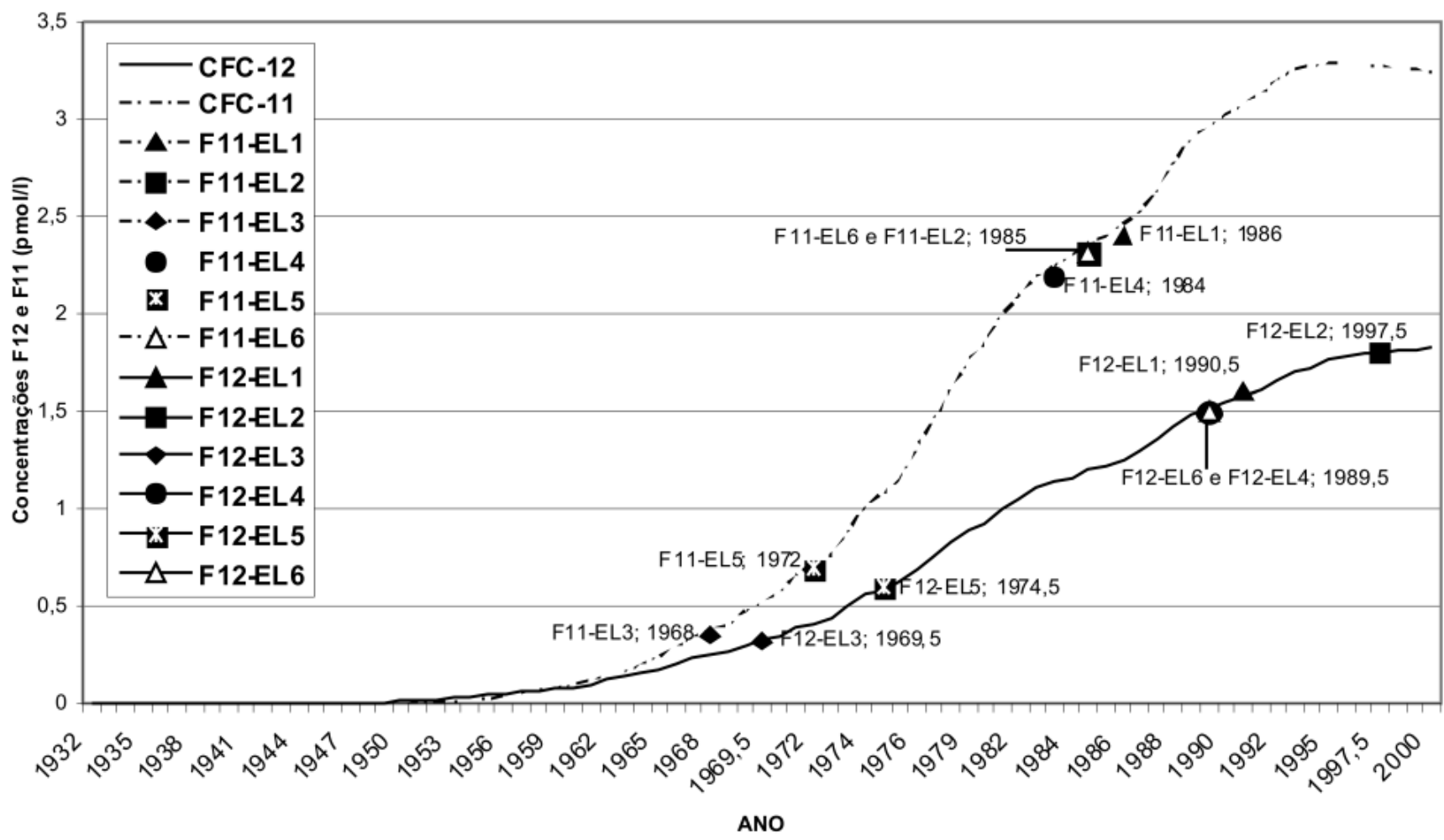

Figura 6 - Gráfico que relaciona as concentrações padrões dos gases $C F C-11$ e CFC-12, quando solúveis em água, com os padrões pontuais resultantes das amostras analisadas (EL2, EL4, EL6 - meio poroso, EL1, EL3, EL5 - meio fraturado). As curva CFC-11 e CFC-12 são os padrões para o hemisfério sul em altitudes de $1000 \mathrm{~m}$ e temperatura média de $20^{\circ} \mathrm{C}$.

que mostraram os resultados mais promissores. A análise desses compostos resulta em idade aparente muito próxima da real, considerando-se que a amostragem foi realizada dentro dos padrões específicos para análise dos gases $\left({ }^{18} \mathrm{O}\right.$, trítio e $\left.\mathrm{CFCs}\right)$, totalmente imersa na mesma água do aquífero para evitar contaminações pelo contato com a atmosfera. Outro fator a ser considerado é que na zona saturada do aquífero não ocorre incremento na concentração de CFCs por agentes externos, o que se pode verificar são as constantes misturas com águas de concentrações variadas nesses elementos, porém essa característica já é considerada no funcionamento dos modelos.

Medidas no sentido de desenvolver uma efetiva gestão das águas subterrâneas da região do Distrito $\mathrm{Fe}$ deral são fundamentais, pois os principais resultados dessa pesquisa mostram que mesmo as águas presentes nas porções mais rasas dos aquíferos freáticos apresentam idades, em média, mais velhas que cinco anos, ou seja, representam misturas de águas recentes com águas que ocuparam o aquífero nos últimos anos. As águas mais profundas apresentam idades mais antigas que 30 anos. Esses resultados mostram a necessidade de se prevenir a contaminação ou sobrexplotação desses mananciais, uma vez que sua renovação total e recuperação pode requerer dezenas de anos.

Agradecimentos Agradecemos aos órgãos de fomento que suportaram o projeto de pesquisa cujos resultados estão em parte apresentados neste artigo, bem como àqueles que subsidiam atuais pesquisas dos autores: $\mathrm{CNPq} /$ FINATEC/Instituto de Geociências - UnB/FUNCAP.

\section{Referências}

Baertschi P. 1976. Absolute ${ }^{18} \mathrm{O}$ content of Standard Mean Ocean Water. Earth and Planetary Science Letters, 31:341.

Busenberg E. \& Plummer L.N. 1993. Use of trichlorofluorocarbon-113 (CFC113) as a hydrologic tracer and age-dating tool for young groundwater. $I n$ : Geological Society of America, 1997 Annual Meeting, Salt Lake City - US, Abstracts with Programs, 29(6):A-78.
Cadamuro A.L.M. 2002. Proposta, avaliação e aplicabilidade de técnicas de recarga artificial em aqüiferos fraturados para condomínios residenciais do Distrito Federal. Dissertação de Mestrado, Instituto de Geociências, Universidade de Brasília, Brasília, 130 p.

Campos J.E.G. \& Tröger U. 2000. Groundwater occurrence in hard rocks in the Federal District of Brasília: A Sustainable Supply? In: Sililo, Groundwater: Past 
Achievements and Future Challenges, I.A.H. Congress, 30, p. 109-113.

Campos J.E.G. \& Freitas-Silva F.H. 1998. Inventário hidrogeológico e dos recursos hídricos superficiais do Distrito Federal. Brasília, IEMA/SEMATEC/UnB, Relatório Técnico Parte IV, 87 p.

Clark I. D. \& Fritz P. (eds.) 1997. Environmental isotopes in hydrogeology. New York, Lewis Publishers, 328 p.

Craig H. 1961. Standard for reporting concentrations of deuterium and oxygen-18 in natural water. Science, 133:1833-1834.

Cunnold D.M., Weiss R. F., Prinn R.G., Hartley D., Simmonds P.G., Fraser P.J., Miller B., Alyea F.N., Porter L. 1997. GAGE/AGAGE Measurements indicating reduction in global emissions of $\mathrm{CCl}_{3} \mathrm{~F}$ and $\mathrm{CCl}_{2} \mathrm{~F}_{2}$ in 1992-1994. Journal Geophysics Research - Atmos. 102(D1):12591269.

Ekwurzel B., Schlosser P., Smethie W.M., Plummer L.N., Busenberg E., Michel L.R., Wepperning R., Stute M. 1994. Dating of shallow groundwater: Comparison of the transient tracers ${ }^{3} \mathrm{H} /{ }^{3} \mathrm{He}$, Chlorofluorocarbons and ${ }^{85}$ K. Water Resources Research. 30(6):1693-1708.

Hageman R., Nief G., Roth E. 1970. Absolute isotopic scale for deuterium analysis of natural waters. Absolute $\mathrm{D} / \mathrm{H}$ ratio for SMOW. Tellus. 22:712-715.

Höhener P., Duwig C., Pasteris G., Kaufmann K., Dakhel N., Harms H. 2003. Biodegradation of petroleum hydrocarbon vapors: laboratory studies on rates and kinetics in unsaturated alluvial sand. Journal Cont. Hydrology, 66:93-115.

International Atomic Energy Agency - IAEA. 1983. Guidebook on nuclear techniques in hydrology. Viena, IAEA, Technical Reports Series, 91, 439 p.

Lousada E.O. 1999. Estudos geológicos e geofísicos aplicados a locação de poços tubulares profundos em aqüiferos fraturados na região do Distrito Federal. Dissertação de Mestrado, Instituto de Geociências, Universidade de Brasília, Brasília, 107 p.
Oster H., Sonntag C., Munnich K.O. 1996. Groundwater age dating with chlorofluorocarbons. Water Resources Research, 32(10):2989-3001.

Schlosser P., Sute M., Sonntag C., Münnich K.O. 1989. Tritiogenic ${ }^{3} \mathrm{He}$ in shallow groundwater. Earth Planet Science Lett, 94:245-256.

Siqueira A.F. 1978. Uso dos dados isotópicos e químicos como indicadores de origem das águas e sais dissolvidos no Aqüifero Calcário Bambuí - Irecê - BA. Tese de Doutorado, Universidade Federal da Bahia, Salvador, 86 p.

Solomon D.K., Schiff S.L., Poreda R.J., Clarke W.B. 1993. A validation of the ${ }^{3} \mathrm{H} /{ }^{3} \mathrm{He}$ method for determining groundwater recharge. Water Resources Research, 29(9):2951-2962.

Souza M.T. 2001. Fundamentos para gestão dos recursos hídricos subterrâneos do Distrito Federal. Dissertação de Mestrado, Instituto de Geociências, Universidade de Brasília, Brasília, 94 p.

Szabo Z., Rice D.E., Plummer L.N., Busenberg E., Drenkard S., Schlosser P. 1996. Age dating of shallow groundwater with chlorofluorocarbons, tritium/helium 3 and flow path analysis, Southern New Jersey Coastal Plain. Water Resource Research, 32(4):1023-1038.

Thompson G.M \& Hayes J.M. 1979. Trichlorofluoromethane in groundwater: A possible tracer and indicator of groundwater age. Water Resource Research, 15(3):546554.

Tolskikhin I.N. \& Kamenskiy I.L. 1969. Determination of ground water age by the $\mathrm{T}-{ }^{3} \mathrm{He}$ method. Geochemistry International, 6:810-811.

Unterweger M.P., Coursey B.M, Shima F.J., Mann W.B. 1980. Preparation and calibration of the 1978 national bureau of standards tritiated waters. International Journal of Applied Radiation and Isotopes, 31:611-614.

Manuscrito ID 10841

Submetido em 10 de março de 2008 Aceito em 02 de fevereiro de 2010 